\title{
Infectious Hospital Agents: A HAI Spreading Simulation Framework
}

\section{Róbert Pethes, Tamás Ferenci, Levente Kovács}

Research and Innovation Center of Óbuda University, Physiological Controls Group, Óbuda University, Budapest, Hungary

Email: pethes.robert@phd.uni-obuda.hu, \{ferenci.tamas, kovacs.levente\}@ nik.uniobuda.hu

\begin{abstract}
Infectious Hospital Agents (IHA) is an individual-based simulation framework that is able to model wide range of infection spreading scenarios in the hospital environment. The simulations are agent-based simulations driven by stochastic events, the evolution of the model is tracked in discrete time. Our aim was to build a general, customisable and extensible simulation environment for the domain of Hospital-Associated Infections (HAIs). The system is designed in Object Oriented fashion, and the implementation is in $C++$. In this paper, the authors describe the motivations and the background of the framework, sketch the conceptual framework, and present a demonstration example.
\end{abstract}

Keywords: Healthcare-Associated Infections; Hospital simulation; Agent-based simulation

\section{Introduction}

Hospital-associated infections (HAI) are infections that patients get while receiving treatment in healthcare settings. In practice, HAIs are often identified as antibioticresistant bacteria, such as Methicillin Resistant Staphylococcus Aureus (MRSA), Clostridium difficile, Drug-resistant Streptococcus pneumonia and Vancomycin Resistant Enterococci (VRE). Antibiotic resistance is the ability of bacteria to resist the effects of an antibiotic [1]. It occurs when bacteria change in a way that reduces the effectiveness of drugs to cure or prevent infections. The molecular mechanism of the resistance development is a complex process, and the most frequent type of resistance is acquired and transmitted via the conjugation of plasmid [2]. Furthermore, the emergence of multidrug-resistant (MDR) bacteria strains increases the seriousness of the problem. According to these facts, it is easy to see that the treatment of these infections are very costly [3] and complicated, that is the reason why prevention gets great emphasis. Surveillance, outbreak investigation and interruption, HAI prevention are included in hospital infection control. In infection control, it is essential to understand the dynamics of infection spreading, and to predict the 
effects of the interventions against HAIs. Mathematical modelling and simulations can help the infection control professionals to improve their understanding of these complex processes, and make better decisions.

Primarily, our aim was to build a simulation framework that is able to simulate a wide range of infection spreading scenarios, but we are faced with the problem, that the spreading process and the infection control processes highly depend on the other hospital processes. Therefore we have to simulate the hospital processes at the same level, what is a difficult problem in itself.

\subsection{Pathogen Sources and Transmission Routes}

In this section, we review the possible pathogen sources and the pathogen transmission routes. Studies have shown that the primary transmission pathway is the patient - healthcare worker $(\mathrm{HCW})$ - patient route $[4,5]$. In other words, the HCWs transmit the pathogens via their hands. Pittet et al. [4] identified the 5 main steps of pathogen transmission via healthcare workers' hands (the pathogen sequence steps) and the evidence supporting each step. The steps are the following:

1. Pathogens are present on the patient's skin or in the patient's immediate environment.

2. Transfer of pathogens to HCW's hands.

3. Pathogens must survive on HCW's hands for at least several minutes.

4. Hand decontamination (hand washing/rubbing or hand antisepsis) by the healthcare worker must be inadequate or omitted entirely.

5. The HCW's contaminated hand(s) must come into direct contact with another patient or with a fomite in direct contact with the patient.

In the prevention of HAI transmission, our aim is to break this sequence. Of course the patient to patient and $\mathrm{HCW}$ to $\mathrm{HCW}$ routes are also important, if we investigate the spread of an airborne diseases, such as influenza. There are some special cases, when we have to consider the patient to patient routes also: when the probability that two patients come into direct contact is not negligible, such as in a pediatric ward. A contaminated environment may also be a source of pathogens in hospitals. For example strains of MRSA can survive and remain viable on dust particles or skin scales for many weeks and months [6], and it is also proven that low densities of MRSA can initiate infections [7]. As we have mentioned in the previous subsection, antibiotic resistant pathogens can emerge caused by the selective pressure of antibiotics, but more commonly, newly admitted patients can carry these pathogens to the hospital $[8,9]$.

\subsection{Infection Control Measures}

Infection control measures are all the interventions against HAIs that a hospital can use to prevent the infections. In this section we briefly overview the most common 
infection control measures:

- Hand hygiene: Obviously, hand hygiene is one of the most important infection control measures $[4,5]$. There are two main factors when we are talking about hand hygiene: compliance and the quality of hand decontamination. In brief, the first one refers to the frequency of hand washing/rubbing, the second refers to the quality of it.

- Staff cohorting: This is a method to restrict the transmission network. If we assign a caregiver to a subgroup of patients, we can eliminate the transmission of the pathogen (via the HCW's hand) between patient groups. In other word, we can decrease the role of the 2nd and 5th steps of the transmission sequence discussed before.

- Patient isolation: This is another way to restrict the transmission network. If a patient is found to be colonized or infected, then isolation is justifiable. In this case, there are special hygiene and precaution rules for the HCW who enters or leaves the room of the isolated patient. However, patient isolation is very costly and often practically impossible as the number of colonized/infected patients are increasing.

- Surveillance: a collection of methods for collecting every information regarding the epidemic process. This can contain a lot of data acquisition methods: swabbing (microbiological sampling) at admission, periodically repeated swabbing of the patients, tracking the patients' temperature chart, illness records, computing the risk factors, etc. The result of the surveillance can be used to ordain special interventions, such as isolation. Recently, there is a new trend to use information technology tools for monitoring hand hygiene compliance and quality [10], and Lehotsky et al. showed that direct, personal feedback can reduce the rate of inadequate hand rubbing [11].

- Patient decolonization: We suppose that patients are persistent carriers after colonization, and this fact has an important role in the pathogen transmission sequence. For example, patients can carry MRSA on their skin, nose or injured skin, therefore they act as a constant source for MRSA transmission [12]. MRSA eradication can be effectively done by using mupirocin and chlorhexidline for decolonization [13].

- Antibiotic usage protocols: antibiotic usage in hospitals has a key role in emergence and spread of HAIs [14], and the applied protocol has a great impact [15].

- Cleaning and sterilization.

\subsection{Hospital Simulations}

The effective operation of hospitals is a key issue for society, and the optimization of the operation of hospitals is an important question in the practical Operational Research/Management Science. The "optimality" has different viewpoints, and the 
resulting healthcare service directly affects the lives of many people. However, hospitals are complex systems, and it is very hard to find an optimal plan to manage hospital resources, and predict the effects of the interventions or the change of the environment. A hospital can be also seen as a queuing system, but the direct use of queuing analytic theory is very hard [16]. One tool, that can help, is computer simulation. A simulation is a simplified replica of a real-world system, and can be used to evaluate "what-if?" scenarios before applying the changes. The most common hospital simulation methods [17] are Discrete Event Simulation (DES), System Dynamics (SD) and Agent-Based Simulation (ABS). DES is applied to model systems that change their states dynamically, stochastically, in discrete time intervals. It is particularly applicable for systems that have queuing structure. System Dynamics is a method of simulating continuous systems. It works on a set of differential equations. In this method, we usually examine cohorts rather than individuals. SD models are more appropriate for studying the interrelationship between elements of the systems. In an Agent-Based Simulation, there are autonomous objects called agents, who are living in an environment and interact with each other.

\subsection{Modelling and Simulations in Infection Control}

The most important model types in HAI modelling and simulation are compartmentbased and agent-based models [18-20]. In a compartment-based model, the population is divided into groups (compartments), and the number of agents of each compartment are tracked in the model. Each compartment represents a stage of the infection history. The most common compartments are Susceptible (S), Exposed (E), Infectious (I) and Recovered or removed (R). Different combinations of these compartments lead to different model structures, and the usual model structures, depending on the aims and the level of details are: S-I, S-I-S, S-I-R and S-I-R-S. Inside a compartment, we suppose homogeneous mixing of the agents. After the compartments are decided, one can define the governing equations of the model therefore the compartment models are given by closed mathematical equations. These equations can be differential or stochastic equations, and since the nature of the system is highly stochastic and the population size in a hospital is relatively low, therefore the latter one is more common. (Compartment models therefore are very similar to System Dynamics discussed in the previous subsection).

In the agent-based simulations of HAI spreading, the agents are patients and HCWs, and the interactions are the treatments. The model is driven by discrete (usually stochastic) events. A model like this can be used to predict the effect of the interventions, and therefore, it can support decision making. In an ABS we can investigate models where the population is inhomogeneous, and we can define any interaction that we can represent with a computer program. Therefore, we have greater flexibility in the modelling compared to the compartment-based models, but this flexibility has a price [21]: the simulation time can be very long due to the complexity, the validation of the model is much more difficult, and in addition, it is very hard to plug an agent-based model into an estimation method to estimate the model parameters from recorded time series. 
In this paper our focus is on agent-based models. Ferrer et al. [22] built a model that combines the operational and the epidemiological perspectives to size-up the effect of understaffing and overcrowding in an Intensive Care Unit (ICU). In their model they have taken into account the work schedule, sick leaves, workload, fatigue and occupation state of HCWs. Milazzo et al. [21] tested the effect of spatial and personnel cohorting. In [23] the spread of influenza like illness was simulated. The model contains the immunity of the patients and the spatiality of the ward (emergency ward), and they tested the effect of infection control policies. Meng et al. [24] built a transmission model based on patient to patient transmission routes, and tested the effect of admission and repeat screening tests, shorter test turnaround time, isolation, and decolonisation. Lee et al. [25] investigated the effect of an MRSA outbreak in a region (Orange Country, California) containing multiple hospitals, they modelled patient movement and the MRSA spreading between the institutions. Hernbeck et al. [26] tracked the motion of HCWs and the patient-HCW, HCW-HCW interactions using sensor network. They have built an agent-based simulation on the resulting hospital society network, and investigated the effect of peripatetic HCWs (having large and diverse set of contacts) on the spread of HAIs.

\section{The Elements of The Conceptual Model}

Our aim was to build an extensible and robust simulation framework to be able to model a wide range of different hospital infection spreading scenarios. These models are infection spreading processes embedded into a hospital simulation. During the design, we have identified, (1) the main hospital processes affecting the infection spreading, (2) then the elements of the infection spreading, and finally (3) the interaction points with each other. The identified processes of (1) - (3) are together what we call conceptual model.

\subsection{Hospital Processes}

The basic organization unit of the hospital is the ward. There are two types of individuals: patients and healthcare workers. We do not deal with visitors, because our primary interests are HAIs (but the model is open: we can extend it with visitors). The patients belong to a specific ward, but the caregivers can work in multiple wards.

The identified hospital processes are the following:

1. The admission process: the arrival of new patients to a hospital ward. New patients can arrive from outside (other hospital, community) or from an another ward of the hospital. Since the characteristic of each ward can be very different. Therefore, the admission pattern of each ward can be different in the same hospital.

2. The discharge of patients: the removal of the patients from the ward. In this framework, we determine (sample from a predefined distribution) the Length- 
of-Stay (LOS) value of each patient at admission, and if the LOS is elapsed, the patient will be removed from the ward. In some cases, the LOS value can change later: for example, if a patent become infected, the LOS value will be increased.

3. Treatment scheduling: every HCW has a list of the treatments that she/he can perform, and at admission, a list of treatments (demand for treatments) is assigned to each patient. Every treatment demand has an urgency value between 0 and 1 (which can change in time). The treatment scheduling process assigns one (or more, if it is necessary) available $\mathrm{HCW}(\mathrm{s})$ to the patients according to the treatments urgency, and also determines the length of the treatment duration (sample from a predefined distribution). The treatment demands list of the patients can also change later, again, in case of infection, the infected patients need more care. In the conceptual model, we do not fix any scheduling method.

4. Treatment processing: The treatment scheduler generates patient-HCW assignments, and assigns a treatment and a treatment duration for each pair. In this step, the HCWs perform the treatment.

\subsection{The Infection Spreading Process}

We suppose that there can be multiple pathogen types in the hospital, and an individual can be clean, colonized or infected for each of the pathogen types. Colonization means that the pathogen's strains are in the different parts of the host's body, but she/he is asymptomatic. In contrast, if somebody is infected, she/he has symptoms, which means sickness. We suppose that patients are reservoirs. Therefore, if a patient becomes colonized, she/he remains colonized (unless we do not do a complete decolonization), but HCWs are not reservoirs, so for HCWs we define maximum colonization time.

We have identified the following elements of the spreading process:

1. Admission colonization: a newly admitted patient can be colonized or infected by one or more pathogens. The admission colonization process decides if an admitted patient is colonized/infected or not.

2. Transmission process: the pathogen transmission from one agent to another. The transmission routes can be: patient to $\mathrm{HCW}, \mathrm{HCW}$ to patent, $\mathrm{HCW}$ to $\mathrm{HCW}$, patient to patient, environment to patient, environment to $\mathrm{HCW}$, patent to environment and HCW to environment. Pathogen transmission or colonization does not mean infection, it means only that the pathogen moves from one agent to another.

3. Infection process: the process when a colonized patient become infected and has symptoms. If a patient becomes infected, then it can increase the LOS and the number of treatment demands.

4. The infection control measures are sub-processes or modifiers of the previ- 
ously defined processes. For example hand washing/rubbing is performed in the treatment processing part, staff cohorting strategy modifies the treatment scheduler, surveillance can be part of almost all of the main processes.

\section{Implementation Issues}

Infectious Hospital Agents is an agent-based programming simulation framework, where the agents are patients and HCWs. The design is in object-oriented fashion and the elements of the sketched conceptual model are implemented via (abstract) classes. We have tried to create a very general and extensible software design, and gave different implementations for each of the elements. These pre-implemented classes can be used as building blocks to set up different simulation scenarios. One can find the details of the object-oriented design in [27]. The implementation is in $\mathrm{C}++$, and for generating differently distributed random numbers, we use the Boost.Random [28] library. We can retrieve all the events, statistics and transmission networks from the implemented event-oriented bookkeeping.

\section{An Example}

Here we present a demonstration simulation example, which is created with the IHA framework. This example is very simple, and some parameter values are not verified, but it can give an insight to the system, and some guidance about the parametrization. We simulate a hypothetical ICU-like ward, with the following properties:

- Only one ward.

- Pathogen: MRSA.

- Simulate only colonization.

- Do not use any infection control measure.

- Only one treatment type with averaged properties.

- Transmission routes: only patient-to-HCW and HCW-to-patient.

- Time unit is minute, time step: 10 minutes.

- The treatment scheduler is a priority based scheduler, where the priorities are the treatment urgency values. If the priorities are the same, it uses random selection.

Model parametrization:

- Admission process: It is a common assumption in hospital simulations, that the admission process is a Poission-process [16]. In this example we use this assumption, and set the admission process to a Poission-process with rate 1/180. Therefore, the mean time between two successive patient arrivals is 
180 minutes.

- The Length-of-Stay distribution: Statistical parameter fitting methods shows that Lognormal, Weibull and Gamma distributions fit best to LOS empirical data [29]. Here we use the lognormal distribution to sample the LOS values of the patients. The lognormal distribution is a continuous probability distribution of a random variable, whose logarithm is normal distributed. The density function of the lognormal distribution is: $\ln \mathscr{N}(x ; \mu, \sigma)=\frac{1}{x \sigma \sqrt{2 \pi}} \exp [-$ $\left.\frac{(\ln x-\mu)^{2}}{2 \sigma^{2}}\right]$, where $\mu$ and $\sigma$ are the mean and standard deviation of the variable's natural logarithm. Setting $\mu=1.3205061822$ and $\sigma=0.3627345555$, we get that $E[L O S]=4$ days and $S D[L O S]=1.5$ days.

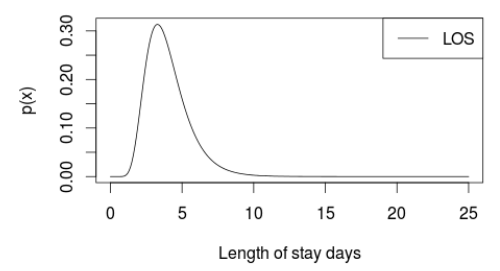

Figure 1

LOS density function

If we plot the probability density function of the LOS values (Figure 1), we can observe that it is an asymmetric heavy tailed distribution, and on the right side of the mean, the decreasing of the plot is not so steep, expressing the fact that greater LOS values may occur with a small, but not negligible probability.

- The probability of admission colonization set to 0.15 according to [30].

- It is a common assumption that the treatment duration is exponentially distributed [16], but it is a huge simplification, and it is hard to find a good parameter value. In this example, we set the parameter value of the exponential distribution to $1 / 10$, expressing that the expected value of the treatment length is 10 minutes. However, this parameter is the weakest part of this example.

- The pathogen transmission probability is set to 0.05 according to [22]. In a more accurate model, the pathogen transmission probability should depend on the contact length, but here, as seen previously, our knowledges about the treatment duration length is inappropriate, therefore we do not use the time dependent model. Using fixed transmission probability, we can ensure that the treatment length does not have a direct effect to the infection spreading process, unless it is not too high.

- Treatment frequency: 8 times a day.

- HCW decolonization period: 1 day. 
The length of the simulation was 120 days, but we have omitted the first 20 days to avoid the initial transients. We have run the simulation independently 100 times, and obtained the result time series by averaging these 100 independent runs: the number of patients in the ward (Figure 2a), the number of colonized patients (Figure 2b), the number of newly colonized patients (Figure 2c) and the rate of the colonized patients (Figure 2d). The number of colonized patients are the number of patients who were colonized at admission plus the number of newly colonized patients. The average number of newly colonized patients for this 100 day period is 17.08 .

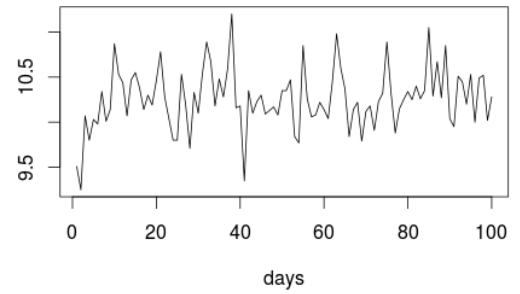

(a)

Average number of patients

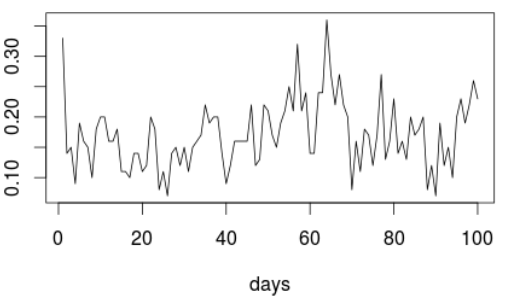

(c)

Average number of newly colonized patients

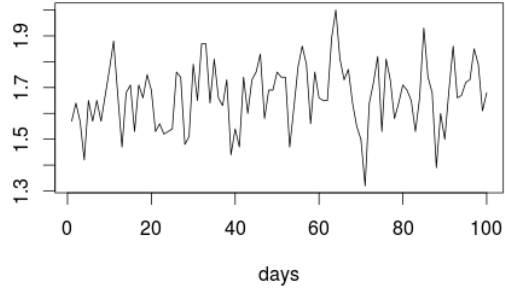

(b)

Average number of colonized patients

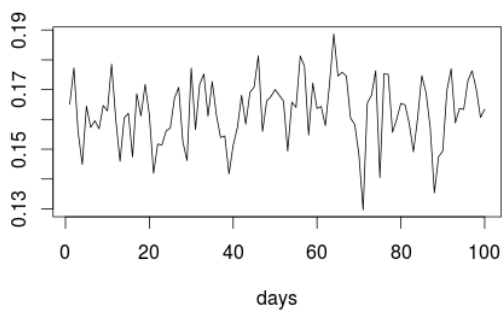

(d)

Rate of colonized patients

Figure 2

The simulation results: averaged time series

In the second part of the example, we study the effect of changing the pathogen transmission probability to the accumulated number of newly colonized patients in the ward. For this reason, we fix every parameter, except the transmission probability, and an $S=\{0.005,0.01,0.02,0.03,0.04, \ldots, 0.15\}$ test value set for the transmission probability. For each $p \in S$, we set the pathogen transmission probability to $p$, ran the simulation 100 times, and computed the average accumulated number of newly colonized patients, and additionally the average accumulated number of colonized patients. The results are summarized in Table 1 and in Figure 3. If $p$ is small, then practically there are no newly colonized patients in the ward, because we as- 
sume that the HCWs are not reservoirs, and the probability that there is a pathogen transmission from a colonized patient to a HCW and the same HCW passes on the pathogen to an another uncolonized patient in a 24 hours length time interval (the maximum colonization time for a $\mathrm{HCW}$ is set to 24 hours) is very small. In this case, the admission colonization process keeps the pathogen in the ward. As we increase the transmission probability, the number of newly colonized patients are increasing (as we expect), and the rate of newly colonized patients is rising in the total number of colonized patients, and about $p=0.11$, the number of newly colonized patients reaches the number of patients who were colonized at admission (supposing that the probability that a patient is colonized at admission is 0.15 ).

Table 1

Results of testing the model in different transmission probabilities

\begin{tabular}{|c|c|c|}
\hline Transmission probability & Colonized patients & Newly colonized patients \\
\hline \hline 0.005 & 154.42 & 0.52 \\
\hline 0.01 & 154.54 & 1.03 \\
\hline 0.02 & 156.83 & 3.85 \\
\hline 0.03 & 158.62 & 6.1 \\
\hline 0.04 & 162.55 & 11.72 \\
\hline 0.05 & 166.78 & 17.08 \\
\hline 0.06 & 175.16 & 28.91 \\
\hline 0.07 & 186.53 & 44.28 \\
\hline 0.08 & 195.66 & 56.92 \\
\hline 0.09 & 211.38 & 75.94 \\
\hline 0.1 & 228.16 & 99.53 \\
\hline 0.11 & 257.84 & 135.36 \\
\hline 0.12 & 279.73 & 162.45 \\
\hline 0.13 & 306.92 & 195.87 \\
\hline 0.14 & 336.84 & 229.11 \\
\hline 0.15 & 358.41 & 255.19 \\
\hline
\end{tabular}

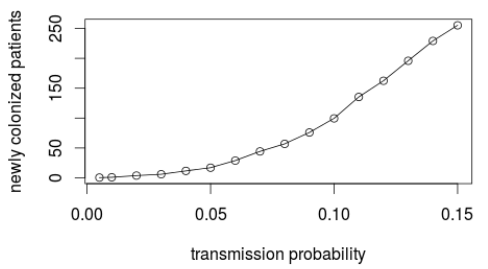

Figure 3

Newly colonized patients in function of transmission probability

Finally we investigate the impact of changing the admission colonization probability parameter to the accumulated number of colonized and newly colonized patients. 
Here, we fix the transmission probability to 0.07 (We use higher transmission probability, to scale up the effect of the change in the admission colonization probability), set the rest of the parameters as before, and test the system setting the admission colonization probability to $\{0.05,0.01,0.02, \ldots, 0.17,0.2\}$. As before, we run the simulations for each of the values of the admission colonization probability 100 times, and obtain the results by averaging the time series. The result are gathered in Table 2 and shown in Figure 4 and Figure 5. We can see in the figures, that the number of colonized patients and the number of newly colonized patients increase more or less linearly when we increase the probability of admission colonization, as it is expected.

Table 2

Results of testing the model against different admission colonization probabilities

\begin{tabular}{|c|c|c|}
\hline Admission colonization & Colonized patients & Newly colonized patients \\
\hline \hline 0.005 & 6.67 & 1.68 \\
\hline 0.01 & 13.06 & 3.68 \\
\hline 0.02 & 26.15 & 7.41 \\
\hline 0.03 & 37.09 & 9.17 \\
\hline 0.04 & 49.1 & 11.06 \\
\hline 0.05 & 60.3 & 13.46 \\
\hline 0.06 & 74.19 & 16.91 \\
\hline 0.07 & 86.52 & 19.89 \\
\hline 0.08 & 100.81 & 24.87 \\
\hline 0.09 & 115.44 & 29.89 \\
\hline 0.1 & 128.77 & 33.53 \\
\hline 0.11 & 140.31 & 36.31 \\
\hline 0.12 & 152.32 & 38.09 \\
\hline 0.13 & 163.93 & 40.44 \\
\hline 0.14 & 176.42 & 43.19 \\
\hline 0.15 & 186.53 & 44.28 \\
\hline 0.16 & 198.63 & 46.55 \\
\hline 0.17 & 209.7 & 47.23 \\
\hline 0.2 & 243.83 & 51.74 \\
\hline & & \\
\hline
\end{tabular}

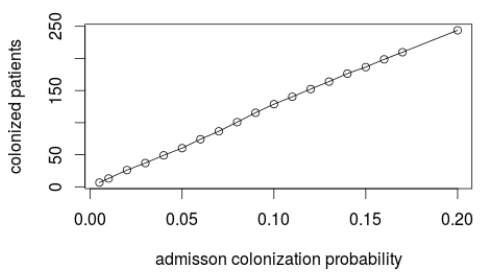

Figure 4

Colonized patients in function of admission colonization probability 


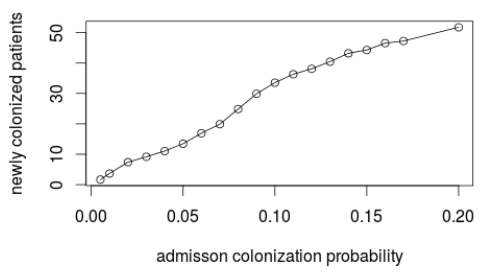

Figure 5

Newly colonized patients in function of admission colonization probability

\section{Discussion}

In this paper we have described the motivations and the background of the IHA simulation framework, presented the conceptual model, and a simple example of the usage of the framework. In the conceptual model, we have identified the main hospital processes affecting the spreading process, we have described the elements of the spreading process, and the way how the hospital processes and the spreading process interact with each other. According to these, we can see, that the spreading process is embedded deeply inside the hospital, and any change of these processes can cause huge change in the other processes. Here, we have excluded a lot of factors from the model, for example: the role of visitors, roster pattern of the HCWs etc., however the conceptual model and the simulation framework are open and extensible. The described work here is a tool development for future research.

\subsection{Future Work}

From software development perspective, we have to work on the validation of our system, because the complexity of the software requires the usage of same systematic software validation method. From modelling perspective, we have to extend the framework with more precise implementation of each sub-processes. Here, we highlight some of them:

- It is a common assumption that the admission of patients is a Poission-process [16]. In practice often that is not the case. When we use Poission-process, we implicitly suppose that the following conditions are true [31]:

1. The probability of more than one arrivals in a short $\Delta t$ length time interval is low $(o(\Delta t))$.

2. The $p(\Delta t)$ probability that at least one patient arrives in a $\Delta t$ time interval is "almost linear" function of $\Delta t,(p(\Delta t)=a \Delta t+o(\Delta t))$.

3. The interarrival times (times between two successive arrivals) are independent random variables.

Here $a$ is a positive constant and $o$ is the common asymptotic notion: $f(x) \in$ 
$o(g(x))$ means $\lim _{x \rightarrow \infty} \frac{f(x)}{g(x)}=0$ The consequence of these assumptions is that the arrival process is a Poission-process. However, as mentioned in [16], these conditions are often not met. For example, sometimes several patients arrive in an Emergency Department (ED) at the same time (several people injured in the same car accident), which clearly violates the condition (1), or the probability of new patient arrivals could depend on the previous arrivals when ED is close to its capacity, which contradicts to condition (3), or the average arrival rate varies during a day, etc. These phenomena may directly effect the infection spread, for example the sudden increase of the load on the HCWs may decrease the hand hygiene compliance, causing higher transmission rate. Consequently, to be able to make the arrival process more precise, we have to create more accurate statistical models, and collect more data to support these models.

- The duration of the patient-HCW contacts is an important factor in the transmission process, since obviously the transmission probability increases with the contact time. However, there is a lack of statistical results about the treatment durations. One way to fix this problem is observing the proximity patterns of the agents in the hospital: collecting data about who is close to whom at what time. This kind of information is invaluable, when we want to study the spreading phenomenon, not only to build statistics about the contact durations. For example, the SocioPatterns project has developed a platform that allows physical proximity measurements using wearable sensors based on radiofrequency identification devices (RFID) [32]. The human body acts as a shield for the radiofrequency signals, therefore the sensors record only contacts when the individuals are facing each other, and thus a contact can be considered as indicative of communication and contact between the individuals. Using these sensors, the temporal proximity networks of patients [33], school children [34], and conference attendees [35] have been successfully recorded.

- If we have statistics concerning the contact durations, we can build and apply different time-dependent transmission models.

- There is a lot of question about the infection process itself. What is the probability, that a colonized patient become infected? Clearly, it depends on not only the pathogen, but also the patient. This probability is very different for a patient in a regular Emergency Department from a patient after immunosuppressive therapy in a transplantation institute. Furthermore, how does the infection increases the LOS of the treatment and treatment demand? These questions lead to the modelling of the immunity of the patients.

\section{Acknowledgement}

The research was supported by European Research Council Starting Grant (ERCStG 679681). Tamás Ferenci was supported by UNKP-16-4/III New National Excellence Program of the Ministry of Human Capacities. 


\section{References}

[1] CDC, Get Smart: Know When Antibiotics Work. http://www.cdc.gov/ getsmart/community/about/antibiotic-resistance-faqs.html.

[2] Alfonso J Alanis. Resistance to antibiotics: are we in the post-antibiotic era? Archives of medical research, 36(6):697-705, 2005.

[3] Eyal Zimlichman, Daniel Henderson, Orly Tamir, Calvin Franz, Peter Song, Cyrus K Yamin, Carol Keohane, Charles R Denham, and David W Bates. Health care-associated infections: a meta-analysis of costs and financial impact on the us health care system. JAMA internal medicine, 173(22):2039-2046, 2013.

[4] Didier Pittet, Benedetta Allegranzi, Hugo Sax, Sasi Dharan, Carmem Lúcia Pessoa-Silva, Liam Donaldson, John M Boyce, et al. Evidence-based model for hand transmission during patient care and the role of improved practices. The Lancet infectious diseases, 6(10):641-652, 2006.

[5] Benedetta Allegranzi and Didier Pittet. Role of hand hygiene in healthcareassociated infection prevention. Journal of Hospital Infection, 73(4):305-315, 2009.

[6] A Rampling, S Wiseman, L Davis, AP Hyett, AN Walbridge, GC Payne, and AJ Cornaby. Evidence that hospital hygiene is important in the control of methicillin-resistant staphylococcus aureus. Journal of Hospital Infection, 49(2):109-116, 2001.

[7] SJ Dancer. The role of environmental cleaning in the control of hospitalacquired infection. Journal of hospital Infection, 73(4):378-385, 2009.

[8] Kepler A Davis, Justin J Stewart, Helen K Crouch, Christopher E Florez, and Duane R Hospenthal. Methicillin-resistant staphylococcus aureus (mrsa) nares colonization at hospital admission and its effect on subsequent mrsa infection. Clinical Infectious Diseases, 39(6):776-782, 2004.

[9] John A Jernigan, Amy L Pullen, Laura Flowers, Michael Bell, and William R Jarvis. Prevalence of and risk factors for colonization with methicillin-resistant staphylococcus aureus at the time of hospital admission. Infection Control \& Hospital Epidemiology, 24(06):409-414, 2003.

[10] Tamás Haidegger, Viktor Varga, Ákos Lehotsky, Péter Róna, Róbert Pethes, Péter Szerémy, László Szilagyi, Tamás Ferenci, and Levente Kovács. Information technology tools employed in infection control. In Computational Intelligence and Informatics (CINTI), 2015 16th IEEE International Symposium on, pages 339-344. IEEE, 2015.

[11] Ákos Lehotsky, László Szilágyi, Tamás Ferenci, Levente Kovács, R Pethes, György Wéber, and Tamás Haidegger. Quantitative impact of direct, personal feedback on hand hygiene technique. Journal of Hospital Infection, 91(1):81-84, 2015. 
[12] Tatiana V Gurieva, Martin CJ Bootsma, and Marc JM Bonten. Decolonization of patients and health care workers to control nosocomial spread of methicillinresistant staphylococcus aureus: a simulation study. BMC infectious diseases, 12(1):302, 2012.

[13] Heidi SM Ammerlaan, Jan AJW Kluytmans, Heiman FL Wertheim, Jan L Nouwen, and Marc JM Bonten. Eradication of methicillin-resistant staphylococcus aureus carriage: a systematic review. Clinical Infectious Diseases, 48(7):922-930, 2009.

[14] Erika MC D’Agata, Pierre Magal, Damien Olivier, Shigui Ruan, and Glenn F Webb. Modeling antibiotic resistance in hospitals: the impact of minimizing treatment duration. Journal of theoretical biology, 249(3):487-499, 2007.

[15] Hong-Rui Sun, Xinxin Lu, and Shigui Ruan. Qualitative analysis of models with different treatment protocols to prevent antibiotic resistance. Mathematical biosciences, 227(1):56-67, 2010.

[16] A Kolker. Queuing analytic theory and discrete events simulation for healthcare: right application for the right problem, 2010.

[17] Murat M Gunal. A guide for building hospital simulation models. Health Systems, 1(1):17-25, 2012.

[18] Hajo Grundmann and B Hellriegel. Mathematical modelling: a tool for hospital infection control. The Lancet infectious diseases, 6(1):39-45, 2006.

[19] Esther van Kleef, Julie V Robotham, Mark Jit, Sarah R Deeny, and William J Edmunds. Modelling the transmission of healthcare associated infections: a systematic review. BMC infectious diseases, 13(1):1, 2013.

[20] Tan N Doan, David CM Kong, Carl MJ Kirkpatrick, and Emma S McBryde. Optimizing hospital infection control: the role of mathematical modeling. Infection Control \& Hospital Epidemiology, 35(12):1521-1530, 2014.

[21] L Milazzo, James L Bown, A Eberst, G Phillips, and JW Crawford. Modelling of healthcare associated infections: a study on the dynamics of pathogen transmission by using an individual-based approach. Computer methods and programs in biomedicine, 104(2):260-265, 2011.

[22] Jordi Ferrer, Maëlle Salmon, and Laura Temime. Nosolink: an agent-based approach to link patient flows and staff organization with the circulation of nosocomial pathogens in an intensive care unit. Procedia Computer Science, 18:1485-1494, 2013.

[23] Marek Laskowski, Bryan CP Demianyk, Julia Witt, Shamir N Mukhi, Marcia R Friesen, and Robert D McLeod. Agent-based modeling of the spread of influenza-like illness in an emergency department: a simulation study. IEEE Transactions on Information Technology in Biomedicine, 15(6):877-889, 2011.

[24] Yang Meng, Ruth Davies, Katherine Hardy, and Peter Hawkey. An application of agent-based simulation to the management of hospital-acquired infection. Journal of Simulation, 4(1):60-67, 2010. 
[25] Bruce Y Lee, Sarah M McGlone, Kim F Wong, S Levent Yilmaz, Taliser R Avery, Yeohan Song, Richard Christie, Stephen Eubank, Shawn T Brown, Joshua M Epstein, et al. Modeling the spread of methicillin-resistant staphylococcus aureus (mrsa) outbreaks throughout the hospitals in orange county, california. Infection Control \& Hospital Epidemiology, 32(06):562-572, 2011.

[26] Thomas Hornbeck, David Naylor, Alberto M Segre, Geb Thomas, Ted Herman, and Philip M Polgreen. Using sensor networks to study the effect of peripatetic healthcare workers on the spread of hospital-associated infections. Journal of Infectious Diseases, page jis542, 2012.

[27] Pethes Róbert, Ferenci Tamás, and Kovács Levente. Infectious hospital agents: an individual-based simulation framework. In Systems, Man, and Cybernetics (SMC), 2016 IEEE International Conference on, pages 3434-3439. IEEE, 2016.

[28] Jens Maurer, D Abrahams, B Dawes, and R Rivera. Boost random number library, 2004.

[29] Alfio Marazzi, Fred Paccaud, Christiane Ruffieux, and Claire Beguin. Fitting the distributions of length of stay by parametric models. Medical care, 36(6):915-927, 1998.

[30] Werner C Albrich and Stephan Harbarth. Health-care workers: source, vector, or victim of mrsa? The Lancet infectious diseases, 8(5):289-301, 2008.

[31] Samuel Karlin and Howard E Taylor. A first course in stochastic processes. Academic press, 1975.

[32] Ciro Cattuto, Wouter Van den Broeck, Alain Barrat, Vittoria Colizza, JeanFrançois Pinton, and Alessandro Vespignani. Dynamics of person-to-person interactions from distributed rfid sensor networks. PloS one, 5(7):e11596, 2010.

[33] Lorenzo Isella, Mariateresa Romano, Alain Barrat, Ciro Cattuto, Vittoria Colizza, Wouter Van den Broeck, Francesco Gesualdo, Elisabetta Pandolfi, Lucilla Ravà, Caterina Rizzo, et al. Close encounters in a pediatric ward: measuring face-to-face proximity and mixing patterns with wearable sensors. PloS one, 6(2):e17144, 2011.

[34] Juliette Stehlé, Nicolas Voirin, Alain Barrat, Ciro Cattuto, Lorenzo Isella, Jean-François Pinton, Marco Quaggiotto, Wouter Van den Broeck, Corinne Régis, Bruno Lina, et al. High-resolution measurements of face-to-face contact patterns in a primary school. PloS one, 6(8):e23176, 2011.

[35] Juliette Stehlé, Nicolas Voirin, Alain Barrat, Ciro Cattuto, Vittoria Colizza, Lorenzo Isella, Corinne Régis, Jean-François Pinton, Nagham Khanafer, Wouter Van den Broeck, et al. Simulation of an seir infectious disease model on the dynamic contact network of conference attendees. BMC medicine, 9(1):1, 2011. 